\title{
Platelet-rich Plasma in Meniscal Repair: Does Augmentation Improve Surgical Outcomes?
}

\author{
Justin W. Griffin MD, Michael M. Hadeed MD, Brian C. Werner MD, \\ David R. Diduch MD, Eric W. Carson MD, Mark D. Miller MD
}

Received: 10 September 2014/ Accepted: 22 January 2015/Published online: 6 February 2015

(C) The Association of Bone and Joint Surgeons \& 2015

\begin{abstract}
Background Increased contact stresses after meniscectomy have led to an increased focus on meniscal preservation strategies to prevent articular cartilage degeneration. Platelet-rich plasma (PRP) has received attention as a promising strategy to help induce healing and has been shown to do so both in vitro and in vivo. Although PRP has been used in clinical practice for some time, to date, few clinical studies support its use in meniscal repair.

Questions/purposes We sought to(1) evaluate whether PRP augmentation at the time of index meniscal repair decreases the likelihood that subsequent meniscectomy will be performed; (2) determine if PRP augmentation in arthroscopic meniscus repair influenced functional outcome measures; and
\end{abstract}

Each author certifies that he or she, or a member of his or her immediate family, has no funding or commercial associations (eg, consultancies, stock ownership, equity interest, patent/licensing arrangements, etc) that might pose a conflict of interest in connection with the submitted article.

All ICMJE Conflict of Interest Forms for authors and Clinical Orthopaedics and Related Research ${ }^{\circledR}$ editors and board members are on file with the publication and can be viewed on request.

Clinical Orthopaedics and Related Research ${ }^{\circledR}$ neither advocates nor endorses the use of any treatment, drug, or device. Readers are encouraged to always seek additional information, including FDA-approval status, of any drug or device prior to clinical use. Each author certifies that his or her institution approved the human protocol for this investigation, that all investigations were conducted in conformity with ethical principles of research, and that informed consent for participation in the study was obtained.

J. W. Griffin, M. M. Hadeed, B. C. Werner,

D. R. Diduch, E. W. Carson, M. D. Miller ( $)$

Department of Orthopaedic Surgery, University of Virginia

Health System, 400 Ray C Hunt Drive, Charlottesville,

VA 22908, USA

e-mail: mdm3p@virginia.edu
(3) examine whether PRP augmentation altered clinical and patient-reported outcomes.

Methods Between 2008 and 2011, three surgeons performed 35 isolated arthroscopic meniscus repairs. Of those, 15 (43\%) were augmented with PRP, and 20 (57\%) were performed without PRP augmentation. During the study period, PRP was used for patients with meniscus tears in the setting of no ACL reconstruction. Complete followup at a minimum of 2 years (mean, 4 years; range, 2-6 years) was available on 11 (73\%) of the PRP-augmented knees and 15 (75\%) of the nonaugmented knees. Clinical outcome measures including the International Knee Documentation Committee (IKDC) score, Tegner Lysholm Knee Scoring Scale, and return to work and sports/activities survey tools were completed in person, over the phone, or through the mail. Range of motion data were collected from electronic patient charts in chart review. With the numbers available, a post hoc power calculation demonstrated that we would have expected to be able to discern a difference using IKDC if we treated 153 patients with PRP and 219 without PRP assuming an alpha rate of 5\% and power exceeding $80 \%$. Using the Lysholm score as an outcome measure, post hoc power estimate was 0.523 and effect size was $-1.1(-2.1$ to -0.05$)$ requiring 12 patients treated with PRP and 17 without to find statistically significant differences at $\mathrm{p}=0.05$ and power $=80 \%$.

Results There was no difference in the proportion of patients who underwent reoperation in the PRP group (27\% [four of 15]) compared with the non-PRP group (25\% [five of 20]; $p=0.89$ ). Functional outcome measures were not different between the two groups based on the measures used (mean IKDC score, 69; SD, 26 with PRP and 76; SD, 17 without PRP; $p=0.288$; mean, Tegner Lysholm Knee Scoring Scale, 66, SD, 32 with PRP and 89; SD, 10 without PRP; $p=0.065$ ). With the numbers available there was no difference in the proportion of patients who returned to 
work in the PRP group (100\% [six of six]) compared with the non-PRP group (100\% [nine of nine]) or in the patients who returned to their regular sports/activities in the PRP group (71\% [five of seven]) compared with the non-PRP group ( $78 \%$ [seven of nine]; $\mathrm{p}=0.75$ ).

Conclusions Patients who sustain meniscus injuries should be counseled at the time of injury about the outcomes after meniscus repair. With our limited study group, outcomes after meniscus repair with and without PRP appear similar in terms of reoperation rate. However, given the lack of power and nature of the study, modest size differences in outcome may not have been detected. Future larger prospective studies are needed to definitively determine whether PRP should be used with meniscal repair. Additionally, studies are needed to determine if PRP and other biologics may benefit complex tear types.

Level of Evidence Level III, therapeutic study.

\section{Introduction}

The meniscus is a critical structure in maintaining the health and function of the knee, but it is often subject to injury. One treatment option for the injured meniscus is partial or total meniscectomy, although well-established data suggest that increased contact stresses occurring long term can lead to poor outcomes in many cases $[2,3,11$, 23-25]. Such findings have led to a renewed focus on meniscal preservation strategies to prevent articular cartilage degeneration. Recent efforts have been directed toward repair or enhancement rather than resection of the injured meniscus $[6,11,13,17,20-22,26]$; however, even with the best techniques, meniscus repairs can fail. Newer options for meniscus transplantation and tissue engineering have shown some promise, but current evidence regarding long-term outcomes compared with meniscus repair is lacking [5, 26]. Recent studies have focused on biologic augmentation techniques to enhance repair healing with the goal of improving patient outcomes [19, 21, 28, 32]. Common methods to enhance vascularity or healing of the meniscus include trephination, synovial abrasion, and application of a fibrin clot.

One possible reason for treatment failure is the relative avascular nature of the meniscus. It is well established that meniscal repair in the setting of anterior cruciate ligament (ACL) reconstruction results in better healing than meniscus repair alone $[30,33,34]$. The vascular supply involves the lateral and medial geniculate arteries that give rise to a plexus, which predominantly supplies the outer third of the meniscus. Meniscal tears within the inner avascular area are prone to poor healing $[18,20]$. Thus, many augmentation techniques are directed toward altering the milieu directly surrounding the meniscus. Biologic enhancement to augment avascular zones of injury has been reported since the 1980s. Recent advances such as fibrin clot, platelet-rich plasma (PRP), and growth factors have been studied and their application to meniscus repair has been evaluated [14, 15, 32].

PRP is derived from a sample of autologous blood prepared until its concentration of platelets is above baseline values [7]. It is theorized that higher levels of platelets can allow for release of growth factors, which may promote angiogenesis and soft tissue healing. The standard for meniscus repair involves an inside-out vertical mattress suture configuration $[17,22]$. The PRP clot can be sutured into the repair site and delivered under direct visualization. Basic science studies have found that PRP used in tendon repair leads to increased forces to failure, larger tendon callus, and increased tenocyte proliferation $[4,7,18,21]$. Additionally, PRP has been demonstrated to enhance meniscal tissue regeneration in vitro and in vivo, as noted in mRNA expression of extracellular matrix proteins compared with meniscal cells without PRP [14]. To date, no studies of which we are aware have investigated clinical use of PRP in meniscal repair enhancement, although it has been a part of clinical practice for some time.

We therefore sought to (1) evaluate whether PRP augmentation at the time of index meniscal repair decreased the likelihood that subsequent meniscectomy; (2) determine if PRP augmentation in arthroscopic meniscus repair influenced functional outcome measures; and (3) examine whether PRP augmentation altered clinical and patient-reported outcomes.

\section{Patients and Methods}

\section{Subjects}

After institutional review board approval was obtained, we completed a retrospective chart review of all patients who underwent arthroscopic meniscus repair at a single institution between February 2008 and December 2011 (Current Procedural Terminology codes 29882 and 29883). Over the 34-month study period, 235 patients were identified who were older than 18 years of age. Exclusion criteria included having had concomitant procedures, including ACL reconstruction or other ligamentous reconstruction (medial collateral ligament, medial patellofemoral ligament) and age younger than 18 years. Patients who underwent partial meniscectomy of the other compartment as well as patients with chondral pathology were included. This resulted in 35 patients who underwent surgery by one of three surgeons (MDM, DRD, EWC) during the study period (Table 1). All 35 patients had radiographic 
evidence of meniscal pathology seen on MRI. Of the 35 patients evaluated, $15(43 \%)$ patients underwent isolated meniscus repair with PRP augmentation and 20 (57\%) patients underwent meniscus repair without augmentation. Subgroup analysis of tear type (simple versus complex) was not possible given the small sample size.

For the PRP group, mean age was 26 years (range, 1946 years) compared with 35 years (range, 19-68 years) in the non-PRP group $(\mathrm{p}=0.045)$. In the PRP group, $73 \%$ were male (11 of 15 ) compared with $85 \%$ (17 of 20 ) in the non-PRP group $(p=0.206)$. In the PRP group, $27 \%$ (four of 15) were smokers compared with $15 \%$ (three of 20) in the non-PRP group $(p=0.794)$. In the PRP group, mean body mass index (BMI) was $24 \mathrm{~kg} / \mathrm{m}^{2}$ ( $\mathrm{SD}, 4 \mathrm{~kg} / \mathrm{m}^{2}$ ) compared with $28 \mathrm{~kg} / \mathrm{m}^{2}\left(\mathrm{SD}, 6 \mathrm{~kg} / \mathrm{m}^{2}\right)$ in the non-PRP group $(\mathrm{p}=0.035)$.

\section{Platelet-rich Plasma Augmentation}

PRP was used by two senior authors (MDM, EWC) during the study period with one of the authors (MDM) performing 17 cases, 13 with PRP, and another author (EWC) performing seven cases, two with PRP. In the group undergoing PRP augmentation, a standardized, commercially available autologous platelet system was used for all

Table 1. Demographic data

\begin{tabular}{lllll}
\hline Characteristic & Overall & With PRP & No PRP & p value \\
\hline Number & 35 & 15 & 20 & $35(14)$ \\
Age (mean years) (SD) & $31(13)$ & $26(9)$ & $11(73)$ & $17(85)$ \\
Sex: male: number (\%) & $28(80)$ & $23.7(4.0)$ & $27.9(6)$ & $3(15)$ \\
BMI (mean $\left.\mathrm{kg} / \mathrm{m}^{2}\right)(\mathrm{SD})$ & $25(6)$ & $4(27)$ & 0.045 \\
Smoker: number (\%) & $7(20)$ & $0(0)$ & 0.206 \\
Diabetes: number (\%) & $0(0)$ & 0.035 \\
\hline
\end{tabular}

$\mathrm{PRP}=$ platelet-rich plasma; $\mathrm{BMI}=$ body mass index; $\mathrm{NA}=$ not available.
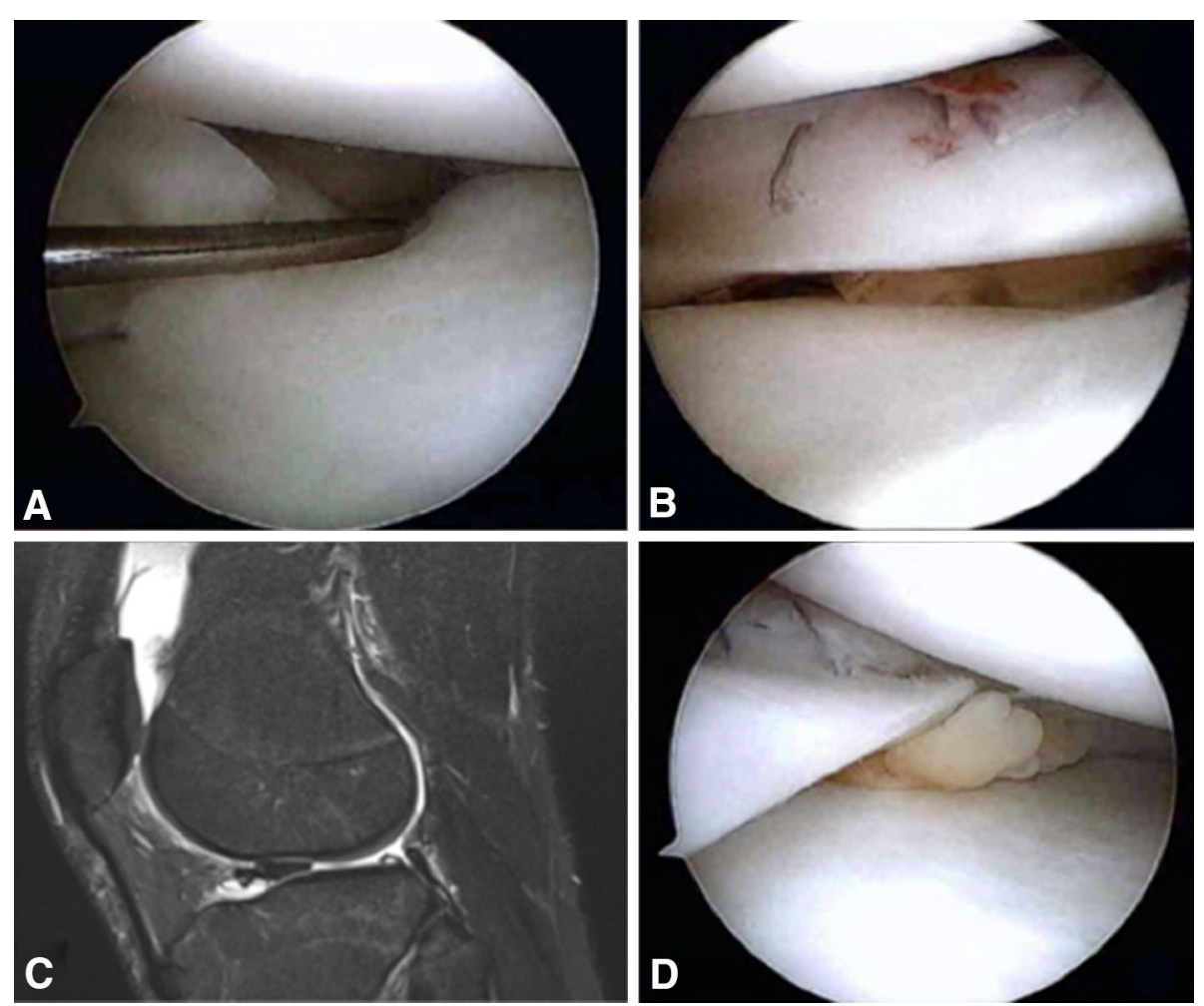

Fig. 1A-D (A) An intraoperative image of a displaced meniscus tear is shown. (B) An inside-out repair is performed with PRP augmentation. (C) MRI demonstrates a bucket-handle lateral meniscus tear and (D) PRP augmentation of this meniscus repair. 
patients (Cascade Platelet Rich Fibrin Matrix, Musculoskeletal Transplant Foundation, Edison, NJ, USA). Patient blood was centrifuged and subsequently, the platelet-rich fibrin matrix was delivered arthroscopically and sutured into the repair site using a PDS suture (Ethicon; Somerville, NJ, USA) in the setting of an inside-out repair (Fig. 1). The postoperative protocol was the same for both the PRP-augmented and the non-PRP group.

\section{Data Collection}

The reoperation endpoint was assessed by chart review. This was confirmed by telephone, by mail, or in person for $15(75 \%)$ patients in the non-PRP group and $11(73 \%)$ patients in the PRP group. Mean followup time for both the PRP-augmented and non-PRP groups was 4 years (range, 2-6 years). Five patients (25\%) in the non-PRP group and four patients $(27 \%)$ in the PRP group were unable to be contacted directly, and their data come exclusively from chart review.

Functional outcome data were collected by telephone, by mail, or in person by one of the authors (MMH) who was not involved in the care of the patients. Those who underwent a subsequent reoperation were not asked to complete the functional outcome scores. Of those patients who did not undergo a subsequent operation, data were collected from $67 \%$ (10 of 15) of the non-PRP group and $64 \%$ (seven of 11) of the PRP group. The functional outcome scores administered included the International Knee Documentation Committee (IKDC) score and the Tegner Lysholm Knee Scale score. With the numbers available, a post hoc power calculation demonstrated that we would have expected to be able to discern a difference using IKDC if we treated 153 patients with PRP and 219 not with PRP assuming an alpha rate of $5 \%$ and power exceeding $80 \%$. Using the Lysholm score as an outcome measure, post hoc power estimate was 0.523 and effect size was $-1.1(-2.1$ to -0.05$)$ requiring 12 patients treated with PRP and 17 without to find statistically significant differences at $\mathrm{p}=0.05$ and power $=80 \%$ (Table 2). We performed a chi-square analysis to determine whether tear type was distributed evenly among the groups (Table 3).
There was no significant difference among the tear type distributions between the groups $(\mathrm{p}=0.39)$.

Clinical outcome data were available for $100 \%$ (20 of 20 ) of patients in the non-PRP group and $80 \%$ (12 of 15) of patients in the PRP group. ROM data collection was completed in clinic by a licensed practitioner involved in the care of the patient. Further patient-reported outcome measures including return to work and return to sports/ baseline activity were administered by telephone, by mail, or in person by one of the authors (MMH) to those patients who had not undergone a reoperation. Data were collected for $67 \%$ (10 of 15) of the non-PRP group and 64\% (seven of 11) of the PRP group. The mean and SD for each group were determined and compared. Return to work and return to sports/baseline activities were rated on a binary scale, and percentages were determined and compared.

\section{Results}

With the numbers available, there was no difference detectable in the proportion of patients undergoing reoperation between the PRP and non-PRP groups. In the PRP group $27 \%$ (four of 15 patients) underwent revision surgery compared with $25 \%$ (five of 20 patients) in the non-PRP group ( $\mathrm{p}=0.89$; Table 2 ). Eight of nine patients who underwent revision surgery after their initial surgery underwent repeat partial meniscectomy with one patient undergoing unicondylar knee arthroplasty. We performed a chi-square analysis to determine whether tear type was distributed evenly among the groups. There was no significant difference among the tear type distributions between the groups ( $\mathrm{p}=0.39$; Table 3$)$.

Of the patients undergoing reoperations, six of nine underwent partial meniscectomy 5 months to 2 years after their index procedure (mean, 1.3 years). Of these patients, two were college athletes (both in the PRP augmentation group) with repeat injuries; two had recurrent pain (both in the non-PRP group), and the others were recreational athletes who had repeat injuries during activity. One of nine patients in the PRP-augmented group underwent revision surgery with partial meniscectomy along with ACL reconstruction after injury during high-level athletics. One

Table 2. Postoperative outcomes

\begin{tabular}{|c|c|c|c|c|}
\hline Measure & Overall & With PRP & No PRP & $\mathrm{p}$ value \\
\hline International Knee Documentation Committee score, mean $( \pm$ SD) & $73( \pm 20)$ & $69( \pm 26)$ & $76( \pm 17)$ & 0.550 \\
\hline Lysholm score, mean $( \pm \mathrm{SD})$ & $80( \pm 23.6)$ & $66( \pm 31.9)$ & $89( \pm 9.7)$ & 0.059 \\
\hline Return to sport $(\%)$ & 80 & 86 & 82 & 0.829 \\
\hline Return to work (\%) & 94 & 100 & 91 & 0.486 \\
\hline
\end{tabular}

$\mathrm{PRP}=$ platelet-rich plasma. 
Table 3. Group tear and repair type characteristics

\begin{tabular}{|c|c|c|c|c|c|}
\hline $\mathrm{R} / \mathrm{L}$ & $\mathrm{M} / \mathrm{L}$ & Tear description & Tear type/location & Repair type & Number of sutures \\
\hline \multicolumn{6}{|c|}{ PRP group } \\
\hline $\mathrm{L}$ & Lateral & Bucket handle and horizontal (posterior horn) & Body and posterior horn & Inside-out & 8 \\
\hline $\mathrm{L}$ & Medial & Peripheral horizontal & Body to posterior horn & Inside-out & 8 \\
\hline $\mathrm{R}$ & Lateral & Peripheral longitudinal & Posterior horn & Inside-out & 6 \\
\hline $\mathrm{R}$ & Lateral & Peripheral longitudinal & Body to posterior horn & Inside-out & 7 \\
\hline $\mathrm{R}$ & Medial & Horizontal & Posteromedial & Inside-out & 6 \\
\hline $\mathrm{R}$ & Lateral & Longitudinal at red-white junction & Body to posterior horn & Inside-out/all inside & 8 \\
\hline $\mathrm{R}$ & Medial & Longitudinal at red-white junction & Body to posterior horn & Inside-out & 4 \\
\hline $\mathrm{L}$ & Medial & Bucket handle & Bucket handle & All inside & 4 \\
\hline $\mathrm{R}$ & Medial & Bucket handle & Bucket handle & Inside-out & \\
\hline $\mathrm{L}$ & Lateral & Peripheral longitudinal & Anterior horn & Outside-in & 3 \\
\hline $\mathrm{R}$ & Medial & Bucket handle & Bucket handle & Inside-out & \\
\hline $\mathrm{L}$ & Medial & Bucket handle & Bucket handle & Inside-out & 9 \\
\hline $\mathrm{L}$ & Lateral & Peripheral longitudinal & Body to posterior horn & Inside-out & 8 \\
\hline $\mathrm{R}$ & Medial & Bucket handle & Bucket handle & Inside-out & 8 \\
\hline $\mathrm{R}$ & Lateral & Vertical & Posterior horn & Inside-out & 6 \\
\hline \multicolumn{6}{|c|}{ Non-PRP group } \\
\hline $\mathrm{R}$ & Lateral & Peripheral longitudinal & Anterior horn & Outside-in & \\
\hline $\mathrm{L}$ & Lateral & Longitudinal & Posterior horn & Outside-in & 1 \\
\hline $\mathrm{L}$ & Lateral & Vertical & Posterior horn & All inside & 1 \\
\hline $\mathrm{R}$ & Lateral & Vertical & Posterior horn & All inside & 3 \\
\hline $\mathrm{R}$ & Lateral & Peripheral longitudinal & Body to posterior horn & All inside & 3 \\
\hline $\mathrm{L}$ & Lateral & Peripheral longitudinal & Bucket handle & Outside-in & 2 \\
\hline $\mathrm{R}$ & Lateral & Bucket handle & Bucket handle & Inside-out & \\
\hline $\mathrm{L}$ & Medial & Bucket handle & Bucket handle & Inside-out & 9 \\
\hline $\mathrm{L}$ & Lateral & Bucket handle & Bucket handle & All inside/outside-in & 6 \\
\hline $\mathrm{R}$ & Medial & Peripheral horizontal & Posterior horn & All inside & 2 \\
\hline $\mathrm{R}$ & Lateral & Longitudinal & Bucket handle & All inside & 4 \\
\hline $\mathrm{L}$ & Lateral & Vertical & Posterior horn & All inside & 2 \\
\hline $\mathrm{R}$ & Medial & Undersurface & Posterior horn & All inside & \\
\hline $\mathrm{L}$ & Lateral & Longitudinal & Posterior horn & All inside & 2 \\
\hline $\mathrm{L}$ & Lateral & Peripheral longitudinal & Posterior horn & All inside & 2 \\
\hline $\mathrm{R}$ & Lateral & Peripheral longitudinal & Posterior horn & All inside & 3 \\
\hline $\mathrm{L}$ & Lateral & Undersurface & Posterior horn & All inside & 1 \\
\hline $\mathrm{R}$ & Medial & Longitudinal & Body to posterior horn & All inside & 3 \\
\hline $\mathrm{L}$ & Medial & Peripheral longitudinal & Body to posterior horn & Inside-out & 4 \\
\hline $\mathrm{L}$ & Medial & Bucket handle & Bucket handle & Inside-out & 6 \\
\hline
\end{tabular}

$\mathrm{PRP}=$ platelet-rich plasma; $\mathrm{R}=$ right $\mathrm{L}=$ left $\mathrm{M}=$ medial; $\mathrm{L}=$ lateral.

patient underwent revision repair of a recurrent torn meniscus at 2 years postindex surgery because of a repeat injury during participation in sports. One 49-year-old patient underwent a unicondylar knee arthroplasty 10 months after his index procedure as a result of persistent pain.

Differences between the groups treated with or without PRP in terms of any of the validated outcomes tools used to evaluate the patients were not apparent with the numbers available. Mean postoperative IKDC score was 76 (range,
46-100) in patients without PRP and 69 (range, 23-94) in patients with PRP augmentation $(\mathrm{p}=0.288)$. The average Lysholm score was 89 (range, 75-100) in patients without PRP and 66 (range, 15-92) in patients with PRP $(\mathrm{p}=0.065)$.

With the numbers available, there were no differences in postoperative ROM, return to work, or return to sport/other baseline activities between the patients treated with and those treated without PRP. Postoperative mean knee 
extension and mean knee flexion measures were $0^{\circ}$ and $125^{\circ}$ (range, $90^{\circ}-135^{\circ}$ ), respectively, for the PRP-augmented group; and $0^{\circ}$ and $119^{\circ}$ (range, $90^{\circ}-130^{\circ}$ ), respectively, for the non-PRP group. Postoperative return to work was $100 \%$ (six of six) in the PRP group and $100 \%$ (nine of nine) in the non-PRP group. Postoperative return to sport/baseline activities was $71 \%$ in the PRP group (five of seven) compared with $78 \%$ (seven of nine) in the nonPRP group $(\mathrm{p}=0.75)$.

\section{Discussion}

The meniscus is critical to the health and function of the knee, yet it is often injured. Recently, there has been an increased effort to determine a more durable alternative to meniscectomy, because the procedure results in higher contact stresses at the articular surface of the knee $[1,2,7$, 15, 16, 23, 25]. Meniscal repairs have been extensively studied but continue to fail for varied reasons. It is thought that the lack of vasculature providing intrinsic nutrition is one reason for poor healing and may explain the increased success seen in concomitant ACL reconstruction [8]. Use of PRP has been shown to augment healing by introducing a higher concentration of various growth factors to the area $[12,14,16,29]$. As noted by Cole et al, the value of PRP use in meniscal repair is the possibility of delivering a local concentration of growth factors and other cytokines directly to the repair site [7]. The appeal of this is to enhance meniscal vascularity. Animal studies have suggested positive effects of PRP augmentation in terms of regenerative effects on meniscal cells in vitro with upregulation of meniscal cell viability and synthesis of sulphated glycosaminoglycans compared with controls [14]. Of note, the cells used in our study were from the inner, typically avascular region of the meniscus.

Studies addressing the beneficial effects of PRP in vitro and in vivo have been published [7, 14, 15] but to our knowledge, no studies concerning the clinical effects of PRP on meniscal repair have been published to date. We therefore sought to evaluate whether PRP augmentation during meniscal repair decreased the likelihood of subsequent meniscectomy, whether PRP augmentation affected validated functional outcome scores, specifically IKDC and Tegner Lysholm Knee Scale scores, and whether PRP affected clinical and patient-reported outcomes, specifically postoperative ROM, return to work, and return to sports/ baseline activity. We found that with the available numbers, there was no difference in any of these outcome measures between those patients who had PRP and those who did not.

There were several limitations to our study. The sample size was one of opportunity and it yielded a small population for analysis. The lack of power analysis made the study underpowered, and thereby we were unable to detect a difference that may, in fact, have been present. To control for as many variables as possible, all meniscal repairs that occurred with concurrent ligamentous operations were eliminated from the sample in an attempt to eliminate confounding factors, but this resulted in a small sample size. A second major limitation was the lack of subgroup analysis to evaluate for differences that may have been present among patient tear types, ages, or demographics. Several studies have suggested that complex or challenging tears are less likely to heal reliably $[6,13,27]$. In this setting, although PRP may have enhanced the biology of the repaired meniscus, failure resulting from tear characteristics may have been inevitable. Further studies are needed to examine PRP augmentation in a prospective manner in matched tear types. Demographic data revealed significant differences between the two groups, which likely affected the outcome analysis. Two important variables, BMI and age, were especially variable between groups. Additional variability was introduced because multiple surgeon outcomes were analyzed in the study. All PRP meniscal repairs were performed by two of the three surgeons involved in the study (MDM, EWC). Meniscal repairs without PRP augmentation were performed by one surgeon during the study period (DRD). One major limitation is that there is no way to be certain that PRP was not used on more difficult tears in the PRP group although both surgeons created a potential selection bias. There is no question that patient age and BMI influenced collagen and cartilage degeneration about the knee, thereby affecting outcomes after meniscus repair. Subtle clinical differences resulting from PRP augmentation may not have been revealed by the outcome measures used.

The outcomes are limited by the survey mailed to patients, and failure was defined only by known recurrence of reoperation. A small number of reoperations in the group lost to followup may change our conclusions and in the absence of followup imaging, it is impossible to determine healing rates in asymptomatic patients. The loss to followup in both groups was similar with slightly more loss to followup in the PRP group. Patients clearly have different thresholds for pursuing revision surgery so as a result our outcome measure is imperfect although clinically important. Postoperative MRI and prospective long-term followup may further elucidate differences between groups. The primary goal of this study was to assess whether PRP augmentation at the time of meniscal repair decreased the likelihood of subsequent meniscectomy. With the numbers available, we found no evidence in support of this claim. In a recent meta-analysis of isolated meniscus repairs, Grant et al found a $17 \%$ failure rate with inside-out repair techniques and an average postoperative 
patient Lysholm score of $87 \%$ [9]. Henning et al demonstrated incorporation of a fibrin clot into an isolated meniscus repair resulted in an $8 \%$ failure rate compared with a $41 \%$ failure rate without the use of fibrin clot [10]. Studies evaluating the PRP effect on meniscal healing in particular are lacking.

Furthermore, with the numbers available, our study found no difference in functional outcomes between patients with and without PRP. No previous studies have evaluated the clinical results of meniscus repair with and without PRP augmentation. There seemed to be a trend toward decreased functional scores in patients treated with PRP; however, the difference was not statistically significant, potentially as a result of the study being underpowered as noted in the post hoc power analysis. Modest-sized differences may not have been detectable given the lack of power. Additionally, tear characteristics, surgeon variability, and demographics were inconsistent between groups. Despite these limitations, all patients were treated for an isolated meniscus tear and a valuable comparison has been made in regard to PRP use, which we hope will encourage future multicenter randomized trials.

Although the theoretic benefits of PRP augmentation in orthopaedic soft tissue healing are possible, the clinical effect has been less clear including our study. There is some evidence for PRP positively influencing in vitro and in vivo healing from animal model studies $[14,15,21,31]$, but no studies to date have examined the clinical consequences of PRP augmentation in isolated meniscal repairs. Despite the limitations of our study, the findings indicate that there may not be an appreciable difference in terms of reoperations and functional outcomes to meniscal repair when PRP is used. Future prospective randomized studies with appropriate sample size to detect a difference will help better elucidate the use of PRP in meniscal healing after meniscus repair.

\section{References}

1. Arno S, Hadley S, Campbell KA, Bell CP, Hall M, Beltran LS, Recht MP, Sherman OH, Walker PS. The effect of arthroscopic partial medial meniscectomy on tibiofemoral stability. Am $J$ Sports Med. 2013;1:73-79.

2. Baratz ME, Fu FH, Mengato R. Meniscal tears: The effect of meniscectomy and of repair on intraarticular contact areas and stress in the human knee. A preliminary report. Am J Sports Med. 1986;4:270-275.

3. Bhatia S, Laprade CM, Ellman MB, Laprade RF. Meniscal root tears: significance, diagnosis, and treatment. Am J Sports Med. 2014;42:3016-3030.

4. Braun HJ, Wasterlain AS, Dragoo JL. The use of PRP in ligament and meniscal healing. Sports Med Arthrosc. 2013;4:206-212.

5. Brophy RH, Matava MJ. Surgical options for meniscal replacement. J Am Acad Orthop Surg. 2012;5:265-272.
6. Choi NH, Kim TH, Son KM, Victoroff BN. Meniscal repair for radial tears of the midbody of the lateral meniscus. Am J Sports Med. 2010;12:2472-2476.

7. Cole BJ, Seroyer ST, Filardo G, Bajaj S, Fortier LA. Platelet-rich plasma: where are we now and where are we going? Sports Health. 2010;3:203-210.

8. de Girolamo L, Galliera E, Volpi P, Denti M, Dogliotti G, Quaglia A, Cabitza P, Corsi Romanelli MM, Randelli P. Why menisci show higher healing rate when repaired during ACL reconstruction? Growth factors release can be the explanation. Knee Surg Sports Traumatol Arthrosc. 2015;23:90-96.

9. Grant JA, Wilde J, Miller BS, Bedi A. Comparison of inside-out and all-inside techniques for the repair of isolated meniscal tears: a systematic review. Am J Sports Med. 2012;2:459-468.

10. Henning CE, Lynch MA, Yearout KM, Vequist SW, Stallbaumer RJ, Decker KA. Arthroscopic meniscal repair using an exogenous fibrin clot. Clin Orthop Relat Res. 1990;252:64-72.

11. Hutchinson ID, Moran CJ, Potter HG, Warren RF, Rodeo SA. Restoration of the meniscus: form and function. Am J Sports Med. 2014;4:987-998.

12. Hutchinson ID, Rodeo SA, Perrone GS, Murray MM. Can platelet-rich plasma enhance anterior cruciate ligament and meniscal repair? J Knee Surg. 2014 Aug 7 [Epub ahead of print].

13. Imade S, Kumahashi N, Kuwata S, Kadowaki M, Ito S, Uchio Y. Clinical outcomes of revision meniscal repair: a case series. Am J Sports Med. 2014;2:350-357.

14. Ishida K, Kuroda R, Miwa M, Tabata Y, Hokugo A, Kawamoto T, Sasaki K, Doita M, Kurosaka M. The regenerative effects of platelet-rich plasma on meniscal cells in vitro and its in vivo application with biodegradable gelatin hydrogel. Tissue Eng. 2007;5:1103-1112.

15. Jang SH, Ha JK, Lee DW, Kim JG. Fibrin clot delivery system for meniscal repair. Knee Surg Relat Res. 2011;3:180-183.

16. Jo CH, Kim JE, Yoon KS, Lee JH, Kang SB, Lee JH, Han HS, Rhee $\mathrm{SH}$, Shin S. Does platelet-rich plasma accelerate recovery after rotator cuff repair? A prospective cohort study. Am J Sports Med. 2011;10:2082-2090.

17. Johnson D, Weiss B. Meniscal repair using the inside-out suture technique. Sports Med Arthrosc. 2012;2:68-76.

18. Kaleka CC, Debieux P, da Costa Astur D, Arliani GG, Cohen M. Updates in biological therapies for knee injuries: menisci. Curr Rev Musculoskelet Med. 2014;3:247-255.

19. Kopf S, Birkenfeld F, Becker R, Petersen W, Starke C, Wruck CJ, Tohidnezhad M, Varoga D, Pufe T. Local treatment of meniscal lesions with vascular endothelial growth factor. J Bone Joint Surg Am. 2010;16:2682-2691.

20. Kurzweil PR, Lynch NM, Coleman S, Kearney B. Repair of horizontal meniscus tears: a systematic review. Arthroscopy. 2014;30:1513-1519.

21. Kwak HS, Nam J, Lee JH, Kim HJ, Yoo JJ. Meniscal repair in vivo using human chondrocyte-seeded PLGA mesh scaffold pretreated with platelet-rich plasma. J Tissue Eng Regen Med. 2014 Jun 19 [Epub ahead of print].

22. Laible C, Stein DA, Kiridly DN. Meniscal repair. J Am Acad Orthop Surg. 2013;4:204-213.

23. Lee SJ, Aadalen KJ, Malaviya P, Lorenz EP, Hayden JK, Farr J, Kang RW, Cole BJ. Tibiofemoral contact mechanics after serial medial meniscectomies in the human cadaveric knee. Am J Sports Med. 2006;8:1334-1344.

24. Lyman S, Hidaka C, Valdez AS, Hetsroni I, Pan TJ, Do H, Dunn WR, Marx RG. Risk factors for meniscectomy after meniscal repair. Am J Sports Med. 2013;12:2772-2778.

25. Muriuki MG, Tuason DA, Tucker BG, Harner CD. Changes in tibiofemoral contact mechanics following radial split and vertical tears of the medial meniscus an in vitro investigation of the 
efficacy of arthroscopic repair. $J$ Bone Joint Surg Am. 2011;12:1089-1095.

26. Noyes FR, Barber-Westin SD. Repair of complex and avascular meniscal tears and meniscal transplantation. J Bone Joint Surg Am. 2010;4:1012-1029.

27. Noyes FR, Barber-Westin SD, Chen RC. Repair of complex and avascular meniscal tears and meniscal transplantation. Instr Course Lect. 2011:415-437.

28. Ra HJ, Ha JK, Jang SH, Lee DW, Kim JG. Arthroscopic insideout repair of complete radial tears of the meniscus with a fibrin clot. Knee Surg Sports Traumatol Arthrosc. 2013;9:2126-2130.

29. Rodeo SA, Delos D, Williams RJ, Adler RS, Pearle A, Warren RF. The effect of platelet-rich fibrin matrix on rotator cuff tendon healing: a prospective, randomized clinical study. Am J Sports Med. 2012;6:1234-1241.

30. Rotterud JH, Sivertsen EA, Forssblad M, Engebretsen L, Aroen A. Effect of meniscal and focal cartilage lesions on patient-reported outcome after anterior cruciate ligament reconstruction: a nationwide cohort study from Norway and Sweden of 8476 patients with 2-year follow-up. Am J Sports Med. 2013;3:535-543.

31. Sheth U, Simunovic N, Klein G, Fu F, Einhorn TA, Schemitsch E, Ayeni OR, Bhandari M. Efficacy of autologous platelet-rich plasma use for orthopaedic indications: a meta-analysis. $J$ Bone Joint Surg Am. 2012;4:298-307.

32. Taylor SA, Rodeo SA. Augmentation techniques for isolated meniscal tears. Curr Rev Musculoskelet Med. 2013;2:95-101.

33. Wasserstein D, Dwyer T, Gandhi R, Austin PC, Mahomed N, Ogilvie-Harris D. A matched-cohort population study of reoperation after meniscal repair with and without concomitant anterior cruciate ligament reconstruction. Am J Sports Med. 2013;2:349-355.

34. Westermann RW, Wright RW, Spindler KP, Huston LJ, MOON Knee Group, Wolf BR. Meniscal repair with concurrent anterior cruciate ligament reconstruction: operative success and patient outcomes at 6-year follow-up. Am J Sports Med. 2014;9:21842192. 